\title{
Lifestyle-Modifikationsprogramm für Menschen mit Diabetes mellitus
}

$>$ Ein Modellprojekt in Kooperation mit dem DVGS e.V. in Düsseldorf

\author{
A. Baldus ${ }^{1}$
}

Mit dem Weltdiabetestag am 24. September 2016 startete in Düsseldorf am Deutschen Diabetes-Zentrum (DDZ) ein Projekt des Regionalen Innovations-Netzwerks RIN in Kooperation mit dem DVGS e.V. Das Projekt wird gefördert durch das Ministerium für Innovation, Wissenschaft und Forschung des Landes Nordrhein-Westfalen. Die wissenschaftliche Begleitung wird gesichert durch das Institut für Sport und Sportwissenschaft der Universität Heidelberg, Professor Dr. Gerhard Huber.

In diesem Projekt wird ein Programm für Menschen mit der Bewegungsmangelkrankheit Diabetes mellitus entwickelt, das schwerpunktmäßig Schulungselemente für Bewegung beinhaltet - aber auch Ernährungsthemata berücksichtigt. Als Zielgruppe wurden primär Menschen mit Diabetes mellitus Typ 2 identifiziert. Voraussetzung für deren Teilnahme ist die Einschreibung in ein Disease-Management-Programm (DMP) bei einem DMPgeschulten Hausarzt oder Diabetologen. Die Finanzierung des Projekts wird anteilig über die Nutzung bestehender Angebote und Versorgungsstrukturen sowie über Partner, Projektmittel und einen Eigenanteil der Teilnehmer erfolgen.

Zusammen mit dem DVGS und dem Verein für Gesundheitssport und Sporttherapie (VGS) Düsseldorf-Ratingen e.V. wurde das DVGS-Zusatzprogramm Bewegung des DVGS als 10-Stunden-Programm weiterentwickelt. Das Bewegungsprogramm wurde bereits durch das Bundesversicherungsamt akkreditiert (also für die Versor-

1 DVGS e.V. gung von Diabetikern, die in DMP-Programme eingeschrieben sind, durch die Krankenkassen anerkannt. Derzeit anerkennt der vdek bundesweit das Programm).

Ein zentraler und neuer Bestandteil im Vorfeld des Bewegungsprogrammes ist die Feststellung des individuellen AktivitätsBedarfs durch den behandelnden DMPArzt. Ärzte der zuständigen Schwerpunktpraxen bzw. Hausarztpraxen in Düsseldorf erheben durch einen evidenzbasierten Ak-

tivitäts-Check das Aktivitätsverhalten der Patienten (sowie den Anteil an sitzender Lebensweise). Die Zuweisung in das Programm erfolgt nach Auswertung der Erhebung. Gemeinsam mit den Ärzten wurden die Voraussetzungen und Zuweisungen sowie die Supervision der Patienten konkretisiert (mit Handreichungen für den Arzt zur Aufklärung über Bewegung bei Diabetes mellitus. Sogenannte Fact-Sheets und Erklärvideos sind derzeit in Konzeption).

\section{Programm-Übersicht}

$>$ Das 10-Stunden-Programm des DVGS ist evidenzbasiert und evaluiert.

$>$ Kontraindikationen werden benannt und sind dem Arzt bekannt.

$>$ Das Bewegungsprogramm ist biopsychosozial konzeptioniert und enthält in jeder Stunde Hinweise zur Bewegung (Bewegungsinhalte, die für die Teilnehmer auch im Alltag und in der Freizeit bzw. am Arbeitsplatz praktizierbar sind), Wissen zum Thema „Bewegung und Diabetes mellitus“ (Wie reduziere ich meinen Langzeitblutzuckerspiegel durch körperliche Aktivität?) und Hinweise zur eigenständigen und nachhaltigen Durchführung individuell angepasster Bewegungsmöglichkeiten (Motivation und Handlungsplanung/Volition).

> Die Qualifikation der durchführenden Sporttherapeuten ermöglicht in der DiabetesGruppe einen individuumsbezogenen Ansatz.

$>$ Eingebunden sind Schrittzähler und Blutzucker-Messgeräte.

\section{Bedarfserhebung (Aktivitätscheck plus Sitzcheck mit Auswertung) und Einweisung durch den Arzt}

$>$ 1. Stunde: 6-Minuten-Gehtest und Einführung in das Thema

$>2$. -4 . Stunde: Bewegungsprogramm

$>$ 5. Stunde: 60 Minuten Bewegung + 60 Minuten Ernährungsinhalte/Themenermittlung für die Wunschstunde 11

$>$ 6.-8. Stunde: Bewegungsprogramm

>9. Stunde: 6-Minuten-Gehtest als Re-Test

$>10$. Stunde: Auswertung und Feedback mit Hinweisen zur weiteren selbständigen Durchführung von Bewegung

$>11$. Stunde: Wunsch-Stunde 
Das Bewegungsprogramm wird im VGS Düsseldorf von hochqualifizierten Bewegungsfachkräften mit Lizenz des DVGS durchgeführt. Es wurde ein expliziter Anteil zum Thema „Ernährung“ eingefügt (in Ergänzung der Patientenschulungsprogramme durch den Arzt) sowie eine „Wunschstunde“ als 11. Stunde. Hier haben die Teilnehmer die Gelegenheit, Fragen zu klären, die ihnen besonders am Herzen liegen.

\section{Ziele des Projekts}

1. Steigerung der Teilnahme von Diabetikern an Bewegungsprogrammen durch Zuweisung durch den Arzt

2. Höhere Arzt-Patienten-Bindung

3. Bindung der Patienten an körperlich aktiven Lebensstil

4. Verbesserung der körperlichen Leistungsfähigkeit und Körperparameter

5. Reduktion des LangzeitblutzuckerWerts

6. Verbesserung der Lebensqualität der Patienten

7. Vermittlung von bewegungsorientierten Gesundheitskompetenzen zum Selbstmanagement für Diabetiker/innen
Online zu finden unter

http://dx.doi.org/

10-1055/s-0042-118755

Verfasser

Angelika Baldus

Geschäftsführerin

DVGS e.V.

Vogelsanger Weg 48

50354 Hürth-Efferen

Tel: 02233-65019

E-Mail: angelika.baldus

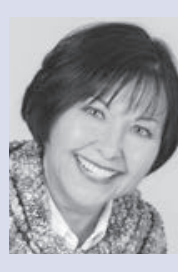

@dvgs.de 